\title{
Clinical management of abnormal Pap tests: differences between US and Korean guidelines
}

\author{
Seyeon Won, Mi Kyoung Kim, Seok Ju Seong \\ Department of Obstetrics and Gynecology, CHA Gangnam Medical Center, CHA University College of Medicine, Seoul, Korea
}

Cervical cancer has been the most common gynecological cancer in Korea but has become a preventable disease with regular screening and proper vaccination. If regular screening is provided, cervical cancer does not progress to more than carcinoma in situ, due to its comparatively long precancerous duration (years to decades). In 2012, the American Society for Colposcopy and Cervical Pathology published guidelines to aid clinicians in managing women with abnormal Papanicolaou (Pap) tests, and they soon became the standard in the United States. Not long thereafter, the Korean Society of Gynecologic Oncology and the Korean Society for Cytopathology published practical guidelines to reflect the specific situation in Korea. The detailed screening guidelines and management options in the case of abnormal Pap test results are sometimes the same and sometimes different in the United States and Korean guidelines. In this article, we summarize the differences between the United States and Korean guidelines in order to facilitate physicians' proper management of abnormal Pap test results.

Key Words: Cervix uteri; Uterine cervical neoplasms; Screening; Papanicolaou test

Received: February 12, 2020 Revised: March 6, 2020 Accepted: March 11, 2020

Corresponding Author: Seok Ju Seong, MD, Department of Obstetrics and Gynecology, CHA Gangnam Medical Center, CHA University, 566 Nonhyeon-ro, Gangnam-gu,

Seoul 06135, Korea

Tel: +82-2-3468-3673, Fax: +82-2-558-1112, E-mail: sjseongcheil@naver.com

Cervical cancer has been the most common gynecological cancer in Korea. Its incidence, however, has been consistently decreasing, from 18.9 per 100,000 females in 1999 to 13.9 in 2016 [1] due to increased interest in cervical cancer screening and the introduction of vaccination [2]. With regular screening and proper vaccination, cervical cancer has become a preventable disease. In fact, if regular screening is provided, cervical cancer does not progress to more than carcinoma in situ, due to its comparatively long precancerous duration (years to decades). In Korea, the promotion and provision of free screening tests for cervical cancer have steadily increased the rate of women undergoing screening [3]. In 2012, the American Society for Colposcopy and Cervical Pathology (ASCCP) published guidelines [4] to aid clinicians' management of women with abnormal cervical cytology and cervical intraepithelial neoplasia (CIN), and they soon became the US standard. Not long thereafter, the Korean Society of Gynecologic Oncology and the Korean Society for $\mathrm{Cy}$ topathology published practical guidelines for early detection of cervical cancer to reflect the high incidence of cervical cancer and low medical costs in Korea [5]. The Papanicolaou (Pap) test is the primary screening method in both guidelines; however, in the case of abnormal Pap test results, the detailed screening guidelines and management options differ in some respects. In this article, we summarize the differences between the US and Korean guidelines in order to facilitate physicians' proper management of abnormal Pap test results.

\section{SCREENING STRATEGIES}

\section{When to start screening?}

According to the guidelines of the ASCCP [4], the Pap test is the standard screening method for cervical cancer and should be started at the age of 21 . Women aged 20 and younger are not considered eligible for screening, regardless of sexual activity, owing to the rarity of cervical cancer cases among that cohort [6]. The ASCCP suggested that the Pap test screening interval for women in their 20s should be 3 years [4]. For such women, spontaneous regression occurs frequently, and the rate of progression 
to invasive cancer is low, notwithstanding the high incidence of human papillomavirus (HPV) infection among that age group [7]. Therefore, HPV triage would be less efficient for those women [8]. In the case of women aged 30 years and older, the screening strategy changes. For them, the ASCCP guidelines call for both a Pap test and an HPV test (i.e., a co-test) every 5 years [4]. The US Preventive Services Task Force (USPSTF) stated that an HPV test alone every 5 years also is possible [9], as women aged 30 years and older are less likely to clear a new HPV infection and more likely to have HPV persistence [10]. Although the cotest may have financial and logistical limitations, it can both increase the detection of prevalent CIN 3 and enhance the detection of adenocarcinoma and adenocarcinoma in situ (AIS) [11].

The Korean guidelines [5] are somewhat different. All women aged 20 years and older who have commenced sexual activity should be screened with a Pap test annually. The co-test for women aged 30 years and older can be applicable. The interval can be extended to every 2 years for a woman who has negative results from both a Pap smear and an HPV test. In summary, the screening test interval is shorter in Korea than in the United States. This difference can be attributed to Korea's lower medical costs and higher cervical cancer incidence rate. Also, whereas in Korea, women aged 20 years and older who have not commenced sexual activity are excluded from screening, in the United States, women aged 21 years and older should be screened regardless of sexual activity.

\section{When to terminate?}

According to the ASCCP [4], screening can be terminated in women aged 65 years and older if three consecutive Pap tests within 10 years are negative or two consecutive co-tests are negative for women with no history of CIN 2 or 3 . Women with a history of CIN 2 or 3 or AIS should be screened for 20 years from the date on which the cervical lesion was treated. Women who have had a total hysterectomy and who have a history of CIN 2 or 3 should be screened every 3 years for a total duration of 20 years from the date on which the cervical lesion was treated. Meanwhile, women who have had a total hysterectomy but lack any history of CIN 2 or 3 can be excluded from screening.

In Korea, screening terminates later than in the United States. Screening can be terminated in women aged 70 years and older if three consecutive Pap smears within 10 years are negative [5].

\section{HPV test alone?}

The HPV test was approved by the Food and Drug Administration (FDA) in 2014 for use in the United States. However, the FDA does not include any specific guidelines for application of this test in cervical cancer screening [12]. The USPSTF stated that screening every 5 years with the HPV test alone can be performed for women aged 30 to 65 years [9]. Also a study of the USPSTF showed that HPV testing detected higher rates of CIN 3 or worse (CIN 3+) and that co-testing did not provide for increased CIN 3 + detection relative to the Pap test [13]. Although the HPV test shows high sensitivity, the efficacy and cost effectiveness of primary HPV screening vary in different medical environments [3]. Considering the Korean situation, which entails lower screening costs and easier access to healthcare, the Pap test, rather than the HPV test, is considered to be the primary screening modality. According to the Korean guidelines [5], the data on primary HPV screening is not yet sufficient to assess its utility for cervical cancer screening [14].

\section{MANAGEMENT OF ABNORMAL PAP RESULTS}

\section{Negative cytology}

We will summarize how to manage negative cytology according to only the ASCCP guidelines [4], as specific corresponding Korean guidelines do not exist. Negative cytology can be classified into three categories. The first is "unsatisfactory cytology" resulting mainly from an insufficiency of squamous cells [15], and its reporting rates are $1.1 \%$ or less [16]. Women with unsatisfactory cytology should undergo a repeat Pap test after 2-4 months. Immediate colposcopy is recommended for women aged 30 years and older who are HPV positive. Women with two consecutive unsatisfactory cytology results should undergo colposcopy. The second category of negative cytology is "negative but absent or insufficient endocervical/transformation component," which means that the squamocolumnar junction may not have been sufficiently collected. Women aged younger than 30 years or 30 years and older with HPV negativity can be returned to routine screening without any further evaluation. However, women aged 30 years and older who are HPV-unknown status should be screened by HPV testing. If women aged 30 years and older are HPV positive, it is recommended that they undergo both repeated Pap testing and HPV testing 12 months later or immediate genotyping of HPV. If HPV 16 or 18 shows positivity by genotyping, immediate colposcopy is recommended. The third category of negative cytology is "negative with a positive HPV test," which presents two options. The first option is repeated co-testing at 12 months, because most cases of HPV infection are cleared rapidly, two-thirds clearing by 12 months [17]. Among a group of women whose HPV infection 
persisted for 1 year, only $21 \%$ progressed to CIN $2+$ within 30 months [17]. If, according to the repeated test, $\mathrm{HPV}$ is positive or the cytology is atypical squamous cells of undetermined significance (ASC-US) or worse, immediate colposcopy is recommended. The second option for treatment of the "negative with a positive HPV test" category is HPV DNA typing. If HPV 16 or 18 is positive, immediate colposcopy is recommended. In Korea, the overall management does not differ from that in the United States, except for the follow-up interval and duration, which are determined at the clinician's discretion.

\section{Atypical squamous cell}

ASC is a common status that is worse than reactive change but does not attain to the criteria of squamous intraepithelial lesion. ASC is classified into ASC-US and high-grade lesion (ASC-H), which runs a high risk of CIN 2+ [18].

\section{ASC-US}

The prevalence rate of ASC-US is 5\%, which is relatively high, though the possibility of its becoming a high-grade lesion such as $\mathrm{CIN} 3+$ is low. In the cases of one-third to two-thirds of women, their ASC-US is not related to HPV infection [8]. Therefore, proactive management, including screening, of women with ASC-US who have a high-grade lesion (e.g., CIN 2+) has proved to be problematic. There are three options for women with ASCUS: repeated Pap test, HPV test, or colposcopy. In the Korean guidelines, any of these options can be chosen, and the actual choice is made by the clinician's personal preference. However, in the ASCCP guidelines, HPV testing is preferred over repeated Pap testing, though colposcopy is not an available option. The more specific guidelines are as follows.

\section{Repeated Pap test}

According to the ASCCP, repeated Pap testing at 12-month intervals can be chosen [4]. In the Korean guidelines [5], the interval of repeated Pap testing is shortened to 6 months. If the result of repeated Pap testing is ASC-US or worse, immediate colposcopy is recommended. If the result is negative, returning to the routine screening program is recommended in the United States, while one additional repeated Pap test (to be performed in 6 months' time) is recommended in Korea. If two consecutive Pap tests show negativity, returning to routine screening is recommended. To sum up, one additional repeated Pap test is needed in Korea, whereas in the United States, the prescription is a return to routine screening.
HPV test

HPV testing is the preferred management for women with ASC-US in the United States. If HPV is detected, immediate colposcopy is recommended. If the HPV test is negative, a return to the routine screening program is recommended.

\section{Colposcopy}

Immediate colposcopy is an option only in Korea. If the result is confirmed as CIN 1 or less, Pap testing at 6 and 12 months or HPV testing at 12 months is recommended. If all of the these results are negative, returning to routine screening is possible. In the United States by contrast, immediate colposcopy, according to the ASCCP guidelines, is not recommended [4]. This may be explained by the significantly expensive medical costs in the United States. Actually, the cost of a colposcopy in the United States is over 230 US dollars, whereas in Korea, it is around a tenth of that (28,950 won), cheaper even than an HPV test (50,890 won) or a Pap test (55,000 won, or free once every two years).

\section{ASC-US in special situations}

The ASCCP guidelines are more subdivided for women aged 21-24 years than is the case in Korea [4], due to the fact that the cervical cancer risk remains low there for women aged under 25 years [6]. An inappreciable difference for women aged 21-24 years is the fact that repeated Pap testing is preferred over HPV testing. If the result of a repeated Pap test is ASC-US, low-grade squamous intraepithelial lesion (LSIL), or negative, again-repeated Pap testing in 12 months is recommended. If the result of a repeated Pap test is ASC-H, atypical glandular cells (AGC), or high-grade squamous intraepithelial lesion (HSIL), immediate colposcopy is recommended. After two consecutive Pap tests are negative, returning to routine screening is possible. If an HPV test is negative once, returning to routine screening is possible. In the Korean guidelines, however, adolescent woman with ASC-US are considered instead of women aged 21-24. For such women, only annual Pap testing is recommended. If the results of a Pap test at 12 months is HSIL or worse, immediate colposcopy should be done. If the results of a Pap test at 24 months are ASC-US or worse, immediate colposcopy should be done.

The management of pregnant women with ASC-US is the same as that of adult women with ASC-US, except for a colposcopy option $[4,5]$. It should be noted that even though colposcopy is permitted for pregnant women, deferring it until 6 weeks after delivery is also acceptable. However, endocervical curettage (ECC) is prohibited during pregnancy. 


\section{ASC-H}

There are no differences in the management of ASC-H between the US and Korean guidelines [4,5]. Colposcopy should be conducted immediately, because the risk of CIN 3+ in women with ASC-H is several times higher than in women with ASC-US or LSIL $[19,20]$. ASC-H, therefore, should be managed in the same manner as HSIL. According to the literature [19], the 5-year cancer risk of women having ASC-H with HPV negativity is approximately $2 \%$, which is too high for conservative observation without further evaluation or treatment. If the result of a colposcopy is not CIN 1, immediate repeated Pap testing and colposcopy can be considered, or, especially according to the Korean guidelines, they can be repeated after 6 months [5]. If two consecutive results of both Pap testing and colposcopy are negative, returning to routine screening is possible. HPV testing for such women is not recommended.

According to the ASCCP guidelines [4], if the result of a colposcopy is CIN 1, co-testing at 1 year is recommended.

\section{ASC-H in special situations}

According to the ASCCP guidelines, immediate colposcopy should be primarily considered for women aged 21-24 years with ASC-H. According to the Korean guidelines, immediate colposcopy should be done for adolescent women with ASC-H. There are no detailed recommendations for pregnant women with ASC-H in either the Korean or ASCCP guidelines.

\section{LSIL}

The natural history of LSIL is clinically equivalent to that of HPV-positive ASC-US [21]. CIN2+ has been found in 11.8\% of woman with LSIL [22]. According to the ASCCP guidelines [4], colposcopy is preferred for women in whom there is LSIL with no HPV test result or in whom there is HPV positivity. For women in whom there is LSIL with HPV negativity, repeated co-testing at 12 months is preferred, or alternatively, colposcopy is acceptable. If the results of co-testing at 12 months is ASCUS or worse or positive for HPV, immediate colposcopy is recommended. If the results of co-testing at 12 months are negative, repeated co-testing at 36 months is recommended.

According to the Korean guidelines [5], immediate colposcopy is preferentially recommended [23] irrespective of HPV test results. ECC should be considered for women in whom cervical lesion is not identified or those for whom colposcopy proves unsatisfactory, except for pregnant women [24].

\section{LSIL in special situations}

For women aged 21-24 years with LSIL, only Pap testing in 12 months is recommended in the ASCCP guidelines [4]; neither colposcopy nor HPV should be considered. For adolescent women with LSIL according to the Korean guidelines [5], observation with Pap testing in 12 months is permitted. Immediate colposcopy is preferred for pregnant women with LSIL according to both the ASCCP and Korean guidelines [4,5]. Deferring of the colposcopy until 6 weeks after delivery is acceptable.

HSIL

There are no specific differences between the ASCCP [4] and Korean guidelines [5] for management of HSIL. The 5-year cancer risk is not statistically different between woman aged 30 to 64 with HPV-negative HSIL and those with HPV-positive HSIL (6.8 and 6.6\%) [18]. Consideration of HPV testing, therefore, is not proper for women with HSIL. Because $40 \%-60 \%$ of women with HSIL turn out to be CIN 2+ by colposcopy [20,22], an immediate excisional procedure can be pursued; otherwise, colposcopy is acceptable. If the colposcopic findings are unsatisfactory, an excisional procedure should be done. If there is no lesion visible by colposcopy, ECC should be done. If the ECC results are negative, two consecutive Pap tests and colposcopy at 6 and 12 months should also be negative in order to return to routine screening. If the finding is CIN 2+ by colposcopy, an excisional procedure is called for. If the colposcopic findingis CIN 1 , there are three options [5]: First, a diagnostic excisional procedure can be performed; second, both the Pap test and colposcopy results can be reviewed once again; and third, both colposcopy and Pap testing should be done at 6 and 12 months until two consecutive results are negative. If the Pap test shows HSIL again, an excisional procedure should be done.

\section{HSIL in special situations}

Only immediate colposcopy (not any excisional procedure) should be considered for adolescent women [5], or those aged 21-24 years (only applicable in United States [4]), with HSIL. Understandably too, only immediate colposcopy is recommended for pregnant women with HSIL as well. However, colposcopic biopsy should be conducted only in cases where high-grade lesion is suspected. Also, ECC is prohibited in pregnant women. If CIN 2 or 3 is found through colposcopic biopsy, an excisional procedure can be delayed until after the birth of the baby, because several studies have shown that high-grade lesion may regress postpartum [25,26]. 


\section{Atypical glandular cells}

AGC as detected on a Pap test may originate from a variety of locations [27]. Therefore, endometrial sampling or ECC might be needed in certain situations. In addition, AGC has not only been associated with benign disease but also with neoplasms of the endometrium, cervix, fallopian tube, and even ovary [28]. According to Katki et al. [18], CIN 3+ was found in $4.7 \%$ and cancer in $2.3 \%$ of woman aged 30 to 64 years with AGC. Basically, immediate colposcopy with ECC is recommended for women with AGC, due to the relatively high risk of $\mathrm{CIN} 3+$. Moreover, HPV testing in concert with colposcopy and ECC should be performed in Korea [5]. Given that endometrial cancer is not HPV associated, routine HPV test is not proper for identification of women who need less invasive management [4]. Endometrial sampling is also recommended in conjunction with colposcopy and ECC in women aged 35 years and older or who are at risk for endometrial cancer. According to the Korean guidelines [5], for women with CIN 1 and negative ECC, a Pap test can be performed at 6 and 12 months or an HPV test can be performed at 12 months. If ASC-US or worse is found in the ensuing Pap test, immediate colposcopy is recommended. Otherwise, a diagnostic excisional procedure is recommended. There are a few differences in the ASCCP guidelines [4]. For women with AGC in whom CIN $2+$ is not found, co-testing at 12 and 24 months is recommended. If both co-tests are negative, co-testing 3 years later is recommended. If any abnormality is found

Table 1. Summary of the United States/Korea differences in screening strategies for cervical cancer

\begin{tabular}{lll}
\hline Screening strategy & \multicolumn{1}{c}{ United States } & \multicolumn{1}{c}{ Korea } \\
\hline Screening age $(\mathrm{yr})$ & $21-65$ & $20-70$ \\
Screening methods & & \\
Pap test & Every $3 \mathrm{yr}$ & Every year \\
HPV test & Every $5 \mathrm{yr}$ at age 30 yr & Not recommended \\
Co-test & Every $5 \mathrm{yr}$ at age 30 yr & Every 2 yr at age 30 yr \\
\hline
\end{tabular}

Pap test, Papanicolaou test; HPV, human papillomavirus; Co-test, Pap test + HPV test. in the subsequent co-test, immediate colposcopy is recommended. Diagnostic excisional procedure is recommended for women with AGC (favor neoplasia) or AIS, regardless of colposcopic results.

\section{AGC in special situations}

Only ASCCP guideline [4] is available. Women aged 21-24 years or those pregnant with AGC should be managed in the same manners as adult women with AGC. Again, ECC and endometrial biopsy are prohibited in pregnant women.

\section{MANAGEMENT OF CIN 1, CIN 2, AND CIN 3}

Abnormal results of Pap test should be managed as described above. Subsequently, once the lesion is revealed to be CIN 1, CIN 2, or CIN 3 by colposcopy or ECC, it should be treated by respective proper methods. CIN 1 has been considered as observable status and known to regress well, and it does not progress frequently to CIN 2+ [29,30]. For women with CIN 1, cotest at 1 year is recommended in the United States [4]. If the subsequent co-test is negative, an age-appropriate test at 3 years (Pap test for women younger than 30 years, co-test for women aged 30 years and older) is recommended. If the age-appropriate test is negative, returning to routine screening is possible. On the other hand, in Korea, Pap testing at 6 and 12 months or HPV testing at 1 year is recommended [5]. If two consecutive Pap tests or HPV tests are negative, returning to routine screening is possible.

CIN 2 and 3 have higher progression rates and lower regression rates than CIN 1. Basically, excisional or ablation therapy, therefore, is recommended for CIN 2 or 3 with adequate colposcopy. However, ablation therapy is not recommended for CIN 2 or 3 with inadequate colposcopy or ECC showing CIN 2, CIN 3, or ungraded CIN. If residual lesion remains at the resection margin after excisional therapy, Pap testing at 6 months or HPV testing at 1 year can be done in follow-up [5].

Table 2. Summary of the United States/Korea differences in management of abnormal Pap tests

\begin{tabular}{|c|c|c|}
\hline Result & United States & Korea \\
\hline ASC-US & HPV test ${ }^{a}$ or Pap test (12 mo) & Pap test (6 mo) or HPV test or colposcopy \\
\hline ASC-H & Colposcopy & Same as the United States \\
\hline LSIL & Co-testa (12 mo, only for HPV-) or Colposcopy & Colposcopy \\
\hline HSIL & Colposcopy or excisional procedure & Same as the United States \\
\hline AGC & Colposcopy and ECC & Colposcopy, ECC and HPV test \\
\hline
\end{tabular}

All procedures should be performed immediately unless otherwise noted.

Pap, Papanicolaou test; ASC-US, atypical squamous cells of undetermined significance; HPV, human papillomavirus; ASC-H, ASC-US and high-grade lesion; LSIL, low-grade squamous intraepithelial lesion; Co-test, Pap test + HPV test; HPV-, women who are HPV negative; HSIL, high-grade squamous intraepithelial lesion; AGC, atypical glandular cells; ECC, endo-cervical curettage.

aPreferred method. 


\section{CONCLUSION}

The overall tendency of the Korean strategy for abnormal Pap test results, relative to the US guidelines, is a shorter interval of follow-up and more invasive evaluation (e.g., immediate colposcopy) rather than following up with HPV or repeated Pap testing [4]. Summarized differences between the guidelines are shown in Tables 1 and 2. These differences reflect the inherent medical environment in Korea. First, the incidence rate of cervical cancer is still higher than in the United States or other developed countries. Second, there is good overall accessibility to medical service in Korea. Third, medical costs are low, and especially, colposcopy is cheaper than a Pap test or HPV test. Strengthening these national advantages and clearly understanding (and consistently following) the treatment guidelines may further reduce the incidence of cervical cancer in Korea.

\section{ORCID}

Seyeon Won: https://orcid.org/0000-0002-2254-2703

Mi Kyoung Kim: https://orcid.org/0000-0002-9520-2339

Seok Ju Seong: https://orcid.org/0000-0003-3820-3412

\section{Author Contributions}

Conceptualization: SJS.

Supervision: SJS.

Writing—original draft: SW.

Writing—review \& editing: SW, MKK, SJS.

\section{Conflicts of Interest}

The authors declare that they have no potential conflicts of interest.

\section{Funding}

No funding to declare.

\section{REFERENCES}

1. Korea Central Cancer Registry, National Cancer Center. Annual report of cancer statistics in Korea in 2016. Sejong: Ministry of Health and Welfare, 2018.

2. Lim MC, Won YJ, Ko MJ, et al. Incidence of cervical, endometrial, and ovarian cancer in Korea during 1999-2015. J Gynecol Oncol 2019; 30: e38.

3. Lim SC, Yoo CW. Current status of and perspectives on cervical cancer screening in Korea. J Pathol Transl Med 2019; 53: 210-6.
4. Massad LS, Einstein MH, Huh WK, et al. 2012 Updated consensus guidelines for the management of abnormal cervical cancer screening tests and cancer precursors. J Low Genit Tract Dis 2013; 17(5 Suppl 1): S1-27.

5. Lee JK, Hong JH, Kang S, et al. Practice guidelines for the early detection of cervical cancer in Korea: Korean Society of Gynecologic Oncology and the Korean Society for Cytopathology 2012 edition. J Gynecol Oncol 2013; 24: 186-203.

6. Benard VB, Watson M, Castle PE, Saraiya M. Cervical carcinoma rates among young females in the United States. Obstet Gynecol 2012; 120: 1117-23.

7. Moscicki AB, Schiffman M, Burchell A, et al. Updating the natural history of human papillomavirus and anogenital cancers. Vaccine 2012; 30 Suppl 5: F24-33.

8. Katki HA, Schiffman M, Castle PE, et al. Five-year risks of CIN 3+ and cervical cancer among women with HPV testing of ASC-US Pap results. J Low Genit Tract Dis 2013; 17(5 Suppl 1): S36-42.

9. US Preventive Services Task Force, Curry SJ, Krist AH, et al. Screening for cervical cancer: US Preventive Services Task Force Recommendation Statement. JAMA 2018; 320: 674-86.

10. Lees BF, Erickson BK, Huh WK. Cervical cancer screening: evidence behind the guidelines. Am J Obstet Gynecol 2016; 214: 43843.

11. Katki HA, Kinney WK, Fetterman B, et al. Cervical cancer risk for women undergoing concurrent testing for human papillomavirus and cervical cytology: a population-based study in routine clinical practice. Lancet Oncol 2011; 12: 663-72.

12. Huh WK, Ault KA, Chelmow D, et al. Use of primary high-risk human papillomavirus testing for cervical cancer screening: interim clinical guidance. Obstet Gynecol 2015; 125: 330-7.

13. Melnikow J, Henderson JT, Burda BU, Senger CA, Durbin S, Weyrich MS. Screening for cervical cancer with high-risk human papillomavirus testing: updated evidence report and systematic review for the US Preventive Services Task Force. JAMA 2018; 320: 687705 .

14. Min KJ, Lee YJ, Suh M, et al. The Korean guideline for cervical cancer screening. J Gynecol Oncol 2015; 26: 232-9.

15. Holton T, Smith D, Terry M, Madgwick A, Levine T. The effect of lubricant contamination on ThinPrep (Cytyc) cervical cytology liquid-based preparations. Cytopathology 2008; 19: 236-43.

16. Moriarty AT, Clayton AC, Zaleski S, et al. Unsatisfactory reporting rates: 2006 practices of participants in the college of american pathologists interlaboratory comparison program in gynecologic cytology. Arch Pathol Lab Med 2009; 133: 1912-6.

17. Rodríguez AC, Schiffman M, Herrero R, et al. Rapid clearance of human papillomavirus and implications for clinical focus on per- 
sistent infections. J Natl Cancer Inst 2008; 100: 513-7.

18. Katki HA, Schiffman M, Castle PE, et al. Five-year risks of CIN 3+ and cervical cancer among women with HPV-positive and HPVnegative high-grade Pap results. J Low Genit Tract Dis 2013; 17(5 Suppl 1): S50-5.

19. Katki HA, Gage JC, Schiffman M, et al. Follow-up testing after colposcopy: five-year risk of CIN 2+ after a colposcopic diagnosis of CIN 1 or less. J Low Genit Tract Dis 2013; 17(5 Suppl 1): S69-77.

20. Massad LS, Collins YC, Meyer PM. Biopsy correlates of abnormal cervical cytology classified using the Bethesda system. Gynecol Oncol 2001; 82: 516-22.

21. Cox JT, Schiffman M, Solomon D, Group A-LTS. Prospective follow-up suggests similar risk of subsequent cervical intraepithelial neoplasia grade 2 or 3 among women with cervical intraepithelial neoplasia grade 1 or negative colposcopy and directed biopsy. Am J Obstet Gynecol 2003; 188: 1406-12.

22. Alvarez RD, Wright TC; Optical Detection Group. Effective cervical neoplasia detection with a novel optical detection system: a randomized trial. Gynecol Oncol 2007; 104: 281-9.

23. ASCUS-LSIL Traige Study (ALTS) Group. A randomized trial on the management of low-grade squamous intraepithelial lesion cytology interpretations. Am J Obstet Gynecol 2003; 188: 1393-400.
24. Partridge EE, Abu-Rustum NR, Campos SM, et al. Cervical cancer screening. J Natl Compr Canc Netw 2010; 8: 1358-86.

25. Vlahos G, Rodolakis A, Diakomanolis E, et al. Conservative management of cervical intraepithelial neoplasia $(\mathrm{CIN}(2-3))$ in pregnant women. Gynecol Obstet Invest 2002; 54: 78-81.

26. Serati M, Uccella S, Laterza RM, et al. Natural history of cervical intraepithelial neoplasia during pregnancy. Acta Obstet Gynecol Scand 2008; 87: 1296-300.

27. Schorge JO, Rauh-Hain JA. Atypical glandular cells. Clin Obstet Gynecol 2013; 56: 35-43.

28. Zhao C, Florea A, Onisko A, Austin RM. Histologic follow-up results in 662 patients with Pap test findings of atypical glandular cells: results from a large academic womens hospital laboratory employing sensitive screening methods. Gynecol Oncol 2009; 114: 383-9.

29. Trimble CL, Piantadosi S, Gravitt P, et al. Spontaneous regression of high-grade cervical dysplasia: effects of human papillomavirus type and HLA phenotype. Clin Cancer Res 2005; 11: 4717-23.

30. Moscicki AB, Shiboski S, Hills NK, et al. Regression of low-grade squamous intra-epithelial lesions in young women. Lancet 2004; 364: 1678-83. 